\title{
Adaptação cultural e validação da versáo portuguesa de Newest Vital Sign
}

\author{
Cross-cultural adaptation and validation of the portuguese version of the Newest Vital Sign \\ Adaptación cultural y validación de la versión portuguesa del Newest Vital Sign
}

Anabela Correia Martins* $\S$

Isabel Maria Andrade** $\S$

$\S$ Contribuiram igualmente

\section{Resumo}

Enquadramento: O Newest Vital Sign (NVS) foi construído para ser um instrumento de administração simples e rápida de avaliação da Literacia em Saúde (LS).

Objetivos: Disponibilizar um instrumento em língua portuguesa para a avaliação de LS da população portuguesa.

Metodologia: Tradução para a língua portuguesa e adaptação cultural da versão original inglesa do NVS. O processo de validação incluiu a análise fatorial e a avaliação das propriedades psicométricas e o estudo da associação com a autoeficácia, Índice de Massa Corporal (IMC), idade e estilos de vida.

Resultados: A fiabilidade foi aceitável, com alfa de Cronbach entre 0,67 e 0,83. Da análise fatorial com rotação ortogonal varimax obteve-se uma solução de dois fatores que explicam $60,97 \%$ da variância. A validade de construção foi ainda suportada pela confirmação das correlações entre a versão portuguesa do NVS (NVS-PT) e a idade, IMC e SES (validade convergente-divergente) e pelo facto de os mais velhos pontuarem mais baixo no NVS-PT $(p=0,001)$.

Conclusão: A versão portuguesa do NVS evidenciou características psicométricas aceitáveis.

Palavras-chave: literacia em saúde; Portugal; população; estudos de validação.

\section{Abstract}

Theoretical framework: The Newest Vital Sign (NVS) test was designed for simple, easy administration on Health literacy (HL) measurement.

Objectives: To make available an instrument in Portuguese to assess the HL level of the Portuguese population.

Methodology: Translation and cross-cultural adaptation of the original English version of the NVS into the Portuguese language. The validation process included the factor analysis and the establishment of its psychometric proprieties and the study of the association with other individual factors, such as the self-efficacy, Body Mass Index (BMI), age and lifestyles. Results: Reliability was acceptable with Cronbach's alpha coefficient between .67 and .83. Using factor analysis with varimax rotation, two subscales explained $60.97 \%$ of the variance. Construct validity was also supported by the confirmation of predefined hypotheses involving expected correlations between the Portuguese version (NVS-PT), BMI, age and SES (convergent-divergent validity). An additional predefined hypothesis was also confirmed with the older subjects obtaining a lower score in the NVS-PT $(p=.001)$.

Conclusion: The NVS-PT evidenced acceptable psychometric characteristics.

Keywords: health literacy; Portugal; population; validation studies.

\footnotetext{
* Ph.D. Professor adjunto, Instituto Politécnico de Coimbra, ESTESC-Dept. Fisioterapia, 3046-854 Coimbra, Portugal [anabelacmartins@estescoimbra.pt]. Morada para correspondência: Rua 5 de Outubro, S. Martinho do Bispo, Apartado 7006, 3046 - 854 Coimbra, Portugal.

* MSc. Professor adjunto, Instituto Politécnico de Coimbra, ESTESC-Dept. Ciências Complementares,3046-854 Coimbra, Portugal [imandrade@estescoimbra.pt].
}

\section{Resumen}

Marco contextual: El Newest Vital Sign (NVS) ha sido diseñado para ser un instrumento de administración fácil y rápida para evaluar la alfabetización en salud (LS, por sus siglas en portugués).

Objetivos: Poner a disposición un instrumento en lengua portuguesa para evaluar el nivel de alfabetización en salud de la población portuguesa.

Metodología: Traducción y adaptación cultural al portugués de la versión original inglesa del NVS. El proceso de validación incluyó el análisis factorial, la evaluación de las propiedades psicométricas y el estudio de la asociación con la autoeficacia, el índice de masa corporal (IMC), la edad y los estilos de vida. Resultados: La fiabilidad ha sido aceptable, con el alfa de Cronbach entre 0,67 y 0,83. Del análisis factorial con rotación ortogonal varimax se obtuvo una solución de dos factores que explican el 60,97\% de la varianza. La validez de constructo ha sido además sostenida por la confirmación de los valores de correlación entre la versión portuguesa del NVS (NVS-PT) y la edad, el IMC y la autoeficacia (validez convergente-divergente) y por el hecho de que los adultos mayores tuviesen una puntuación más baja en el NVS-PT ( $p=0,001)$.

Conclusión: La NVS-PT ha mostrado propiedades psicométricas aceptables.

Palabras clave: alfabetización en salud; Portugal; población; estudios de validación.

Recebido para publicação em: 09.04.13

Aceite para publicação em: 26.05 .14 


\section{Introduçáo}

O conceito de Literacia em Saúde (LS) traduz a evolução no processo de definição, redefinição e quantificação dos níveis de literacia funcional em saúde da população adulta. Ao mesmo tempo, houve o reconhecimento da necessidade de competências sofisticadas em literacia para viver em sociedade, e que um baixo nível de literacia pode ter um efeito negativo sobre a saúde e cuidados de saúde (Berkman, Davis, \& McCormack, 2010).

A LS é cada vez mais vista como um determinante-chave em saúde, sendo crescente a evidência de que um baixo nível de LS está associado a taxas elevadas de morbidade e mortalidade. Um nível inadequado de LS pode traduzir-se por dificuldades na comunicação com os profissionais de saúde, criar barreiras na gestão da doença crónica, reduzir a probabilidade de comportamentos de prevenção da doença, incrementar a ocorrência de erros de medicação, potenciar o risco de hospitalização e pode ainda acarretar uma menor qualidade de vida (Eichner \& Dullabh, 2011).

Vários estudos evidenciam que os níveis de LS são baixos em grupos de indivíduos socialmente desfavorecidos; estes mesmos grupos exibem taxas elevadas de doenças não comunicáveis e evitáveis, para além de que têm maior dificuldade em ter acesso a serviços de saúde e em gerir a sua própria saúde. Assim, a melhoria do nível de LS foi identificada pela Organização Mundial de Saúde (OMS) como uma estratégia-chave para potenciar a saúde de populações vulneráveis e para reduzir desigualdades em saúde, quer em países desenvolvidos quer em desenvolvimento (Organização Mundial de Saúde, 2009).

Portugal carece de uma avaliação do nível de LS da população. Mais ainda, a maior parte dos instrumentos existentes utilizados para medir a LS estão em língua inglesa, pelo que existe a necessidade de obter um instrumento de medida adequado em língua portuguesa. O objetivo deste estudo foi assim obter um instrumento em língua portuguesa, de administração rápida e fiável, para a avaliação de níveis de LS na população portuguesa, e ao mesmo tempo adequado para utilização ao nível dos cuidados de saúde primários.

A nossa escolha recaiu sobre o Newest Vital Sign (NVS) que mede a LS funcional, com base num rótulo nutricional e que é acompanhado de seis questões, requerendo entre três a cinco minutos para a sua administração. Dado que a versão original está em língua inglesa, foi necessário proceder à tradução para português, retroversão, adaptação cultural e validação para a população portuguesa.

\section{Enquadramento}

A LS engloba os conhecimentos e as capacidades dos indivíduos para poderem fazer face às exigências em saúde da sociedade moderna, por isso, a avaliação do nível de LS poderá contribuir para uma maior perceção das aptidões individuais em matéria de saúde, ajudar a adequar a informação em saúde ao público-alvo num formato que estes entendam, e sobre o qual possam atuar no dia a dia.

Pese embora o reconhecimento crescente da importância da LS, a falta de consenso no que à definição diz respeito ou às suas dimensões conceptuais, limita as possibilidades de quantificação e comparação. Recentemente, Sorensen et al. (2012) publicaram uma revisão sistemática da literatura que incluía 17 definições e 12 modelos conceptuais de LS: desta revisão resultou uma definição integrada de LS e um modelo conceptual incluindo dimensões baseadas na evidência relativas ao conhecimento, à motivação e às competências necessárias para o acesso, compreensão, avaliação e utilização de informação/recursos em saúde, ao nível dos cuidados, da prevenção da doença e da promoção de saúde.

$\mathrm{Na}$ Europa, pela primeira vez, a avaliação e comparação do nível de LS foi levada a cabo no âmbito do European Health Literacy Project 20092012 desenvolvido pelo consórcio European Health Literacy Survey Consortium (HLS-EU Consortium), em oito países: Áustria, Bulgária, Alemanha, Grécia, Irlanda, Países Baixos, Polónia e Espanha. Os resultados mostraram que aproximadamente um em cada dois participantes, em média 47\%, evidencia risco de limitada $\mathrm{LS}$, para além de que os níveis variam entre os países intervenientes. Em certos subgrupos populacionais o risco é mais elevado, tais como idosos, indivíduos com baixo nível de educação formal, com baixo rendimento económico e que consideram ter uma condição de saúde deficitária (European Health Literacy Survey Consortium, 2012). 
Sorensen et al. (2012) apontam ainda que um indivíduo com um nível adequado de LS tem as competências necessárias para poder ser responsabilizado pela sua própria saúde, assim como pela saúde da sua família e/ou comunidade.

À semelhança da LS, o conceito de autoeficácia é também considerado um construto ao nível individual: centra-se na teoria social cognitiva de Bandura, enfatizando o papel da aprendizagem por observação, da experiência social e do determinismo recíproco no desenvolvimento da personalidade. Segundo o mesmo autor, as atitudes de um indivíduo, as suas competências e aptidões cognitivas desempenham um papel major na forma como são percecionadas as vivências e no desempenho comportamental face a diferentes situações. A autoeficácia é assim uma componente essencial do autoconceito. Aqueles que evidenciam uma elevada autoeficácia visualizam cenários de sucesso que lhes garantem orientação, proporcionam um desempenho adequado, traduzindo-se em resultados positivos (Bandura, 1997).

Em matéria de educação para a saúde ou promoção da saúde, a avaliação da autoeficácia tem sido considerada um fator-chave para obter melhores resultados em saúde (Pajares, 2002). Diferentes níveis de autoeficácia podem promover ou limitar a motivação para agir; uma elevada autoeficácia está associada a um melhor estado de saúde, maior participação social e mais responsabilidades (Bandura, 1997).

Embora um baixo nível de LS possa existir em todas as populações, é mais comum nos idosos (Barrett, Puryear, \& Westpheling, 2008). Paralelamente, estes caracterizam-se por um maior número de doenças crónicas e recorrem mais vezes aos serviços de cuidados de saúde, para além de que o seu estado físico e cognitivo pode dificultar o acesso e o uso de informação adequada em saúde (Federal Interagency Forum, 2008).

Numa meta-análise recente, Dewalt, Boone, e Pignone (2007) apontaram uma associação entre LS limitada e o aumento da taxa de hospitalização, menor recurso a medidas preventivas em cuidados de saúde e a adesão terapêutica inadequada.

Ainda, segundo dados da American Medical Association Foundation, existe evidência de que os indivíduos com baixa LS correspondem àqueles menos capazes de implementar medidas preventivas em saúde, podendo os custos estimados desse baixo nível de LS na economia dos Estados Unidos da América (EUA) ser superiores a 73 biliões de dólares/ ano (White, 2008).

Assim, considerando a LS um determinante-chave em saúde, o objetivo deste estudo foi o de obter uma versão em língua portuguesa de um instrumento de avaliação da LS funcional, de administração rápida e simples, adequado aos cuidados de saúde primários, e analisar a associação com a autoeficácia, fatores individuais comportamentais e estilos de vida.

\section{Metodologia}

\section{Adaptaçáo cultural}

A tradução de um instrumento de medida para uma outra língua exige diferentes níveis de equivalências, quer de natureza lexical (linguagem) quer cultural.

A versão portuguesa do NVS (NVS-PT) foi desenvolvida através de um procedimento de tradução-retroversão, em conformidade com as recomendações para a tradução de questionários propostas pelo Scientific Advisory Committee of the Medical Outcomes Trust (2002).

As autoras da versão portuguesa, nativas da língua portuguesa, com elevado nível de fluência na língua inglesa, traduziram de forma independente o rótulo nutricional e a folha de pontuações com seis questões, que constituem a versão original do NVS, em inglês dos EUA. Ambas as traduções foram analisadas no seio de um primeiro painel de consenso. A versão de consenso obtida foi ainda alvo de avaliação de conformidade no que diz respeito à regulamentação europeia/portuguesa em matéria de rotulagem nutricional. Esta versão preliminar foi sujeita a retroversão cega para a língua inglesa por dois especialistas bilingues independentes. Foram analisadas e tratadas todas as inconsistências entre a versão inglesa obtida e a versão original por um segundo painel de consenso, para assim garantir a obtenção de um instrumento compreensível e concetualmente consistente com o original. Embora garantindo a consistência com o instrumento original, algumas expressões ou frases foram ligeiramente alteradas de forma a poderem ser compreendidas na íntegra pela população portuguesa.

Foi realizado um pré-teste da versão preliminar do NVS-PT a um grupo piloto de dez indivíduos com o objetivo de identificar eventuais dificuldades 
de interpretação decorrentes da tradução. Após o preenchimento, os respondentes foram questionados acerca de cada um dos itens (i.e. se sentiram dificuldade na compreensão de algum item).

\section{Procedimento de validação}

\section{Amostra}

O processo de validação integrou duas amostras. A Amostra 1 incluiu estudantes do $1^{\circ}$ ao $4^{0}$ ano de licenciatura de todas as áreas científicas da Escola Superior de Tecnologia da Saúde de Coimbra, bem como da Escola Superior de Educação, do Instituto Politécnico de Coimbra. Correspondeu a um total de 457 estudantes, com idades compreendidas entre os 18 e os 50 anos $(M=20,9 ; \mathrm{DP}=4,4)$. A Amostra 2 englobou homens e mulheres recrutados num Centro de Dia e numa Universidade Sénior, incluindo 78 participantes, com idades entre os 45 e os 94 anos $(\mathrm{M}=69,82 ; \mathrm{DP}=9,16)$. A razão para existirem estas duas amostras prende-se com o facto de estarmos perante grupos etários extremos, destacados na literatura como evidenciando diferentes níveis de literacia em saúde.

O estudo foi aprovado pelas instituições e decorreu de acordo com os princípios éticos da Declaração de Helsínquia. Os participantes deram o seu consentimento informado.

\section{Medidas de validaçáo}

Todas as medidas usadas na análise da validade do construto do NVS-PT foram aquelas incluídas no protocolo do estudo, tendo sido obtidas concomitantemente à aplicação do NVS-PT. A caracterização demográfica e clínica englobou estilos de vida/comportamentais (dieta saudável e atividade física) e o Índice de Massa Corporal (IMC). A autoeficácia foi avaliada com base na versão portuguesa da Escala de Autoeficácia (Self-Efficacy Scale, SES) (Ribeiro, 1994), que contém 15 itens, com base numa escala tipo Likert de 7 pontos (1Discordo fortemente a 7 - Concordo fortemente). A pontuação final é a soma dos 15 itens, variando entre 15 e 105, correspondendo maiores pontuações a uma autoeficácia mais elevada. A versão original evidenciou propriedades psicométricas satisfatórias, e fidelidade (alfa de Cronbach $=0,84$ ) e validade adequadas.

\section{Análise estatística}

A descrição das variáveis para a caracterização demográfica e clínica foi realizada através de tabelas de frequências, médias e Desvios-Padrão (DP). Análise de Variância Univariada (ANOVA) foi usada para explorar as diferenças entre os subgrupos.

De acordo com as recomendações de Barrett, Smith, e Wellings (2004), a pontuação em falta (missing) em um ou dois itens da SES foram substituídos pelo valor médio dos itens a que os sujeitos haviam respondido. Dados em falta sobre variáveis sociodemográficas e estilos de vida foram irrelevantes e aleatórios e, portanto, não substituídos.

Para avaliar a consistência interna do NVS-PT calculouse o coeficiente alfa de Cronbach, as correlações itemtotal e valores de alfa quando o item foi eliminado. Nunnaly e Bernstein (1994) indicam 0,70 como um valor aceitável de coeficiente de confiabilidade, mas limiares mais baixos são por vezes utilizados na literatura. Para esses autores, a correlação item-total de 0,30 ou superior foi considerado aceitável para cada item nas subescalas.

Apesar da versão original apresentar apenas um domínio, a validade de construto foi estudada através de análise fatorial exploratória para testar a hipótese que os seis itens do questionário estão representados em dois fatores. Os itens com cargas fatoriais e comunalidades $\geq 0,40$ foram considerados aceitáveis (Staquet, Hays, \& Fayers, 1998). Simultaneamente, a validade de construto foi avaliada por meio de correlações com medidas forte (convergente) ou fracamente (divergente) relacionadas, de acordo com as expetativas baseadas nos construtos teóricos (Scientific Advisory Committee of the Medical Outcomes Trust, 2002).

A validade convergente e a validade divergente foram analisadas por meio de correlações entre os itens e a escala total, inter-itens e com a SES, IMC e idade, por meio do coeficiente de correlação de Pearson. Colocaram-se as hipóteses que as pessoas mais velhas, com IMC mais elevados e com menor pontuação SES teriam menor pontuação no NVS-PT.

Para o teste de hipóteses, uma avaliação prévia das premissas das variáveis métricas foi considerada: para a simetria foi utilizado o coeficiente de Skewness e o achatamento da distribuição foi testado através do coeficiente de kurtose. Para avaliar a adesão à distribuição normal, foram utilizados os testes não paramétricos de Kolmogorov-Smirnov (fator de 
correção de Lilliefors) para amostras superiores a 50 indivíduos e/ou Shapiro-Wilk para amostras iguais ou inferiores a 50 indivíduos.

Para reforçar a análise da validade do NVS-PT, as diferenças entre sexo e faixa etária $(\geq 65$ anos e $<65$ anos de idade) foram analisadas utilizando o teste $t$ de Student e as diferenças entre os estilos de vida/ comportamentais (dieta saudável e atividade física) foram analisados por meio de análise de variância com o teste de Scheffe para comparações múltiplas, quando mais de dois grupos estavam presentes. Quando não se assumiu homogeneidade de variâncias (teste de Levene), o teste de Brown-Forsythe (teste robusto de igualdade de valores médios) foi aplicado, e o teste de Kruskal-Wallis não paramétrico, quando os dados não seguiam uma distribuição normal. A interpretação estatística foi realizada com base no nível de significância de $\mathrm{p}<0,05$. Para o tratamento dos dados usou-se o Statistical Package for the Social Sciences (SPSS), versão 19.

\section{Resultados}

\section{Adaptação cultural}

A segunda versão preliminar do NVS-PT teve boa aceitação no pré-teste. Em geral, houve uma correta compreensão dos itens e não foram reportadas dificuldades no preenchimento do questionário. A versão final do NVS-PT foi obtida num terceiro painel de consenso, a qual foi usada no estudo de validação (ver Figura 1).

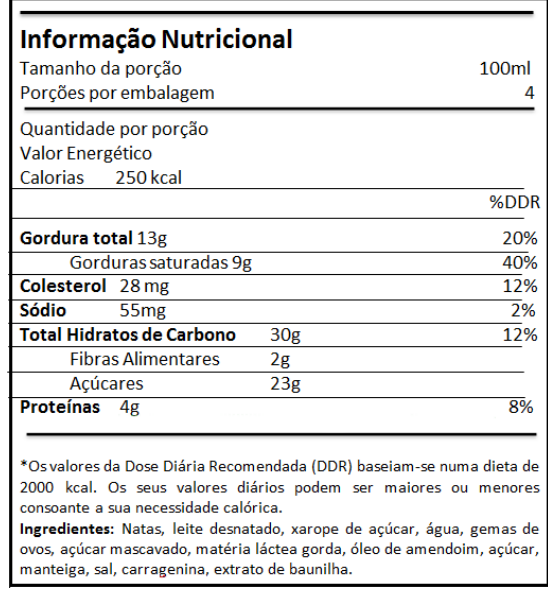

Figura 1. Versão Portuguesa do Newest Vital Sign (NVS-PT).

\section{Interpretação}
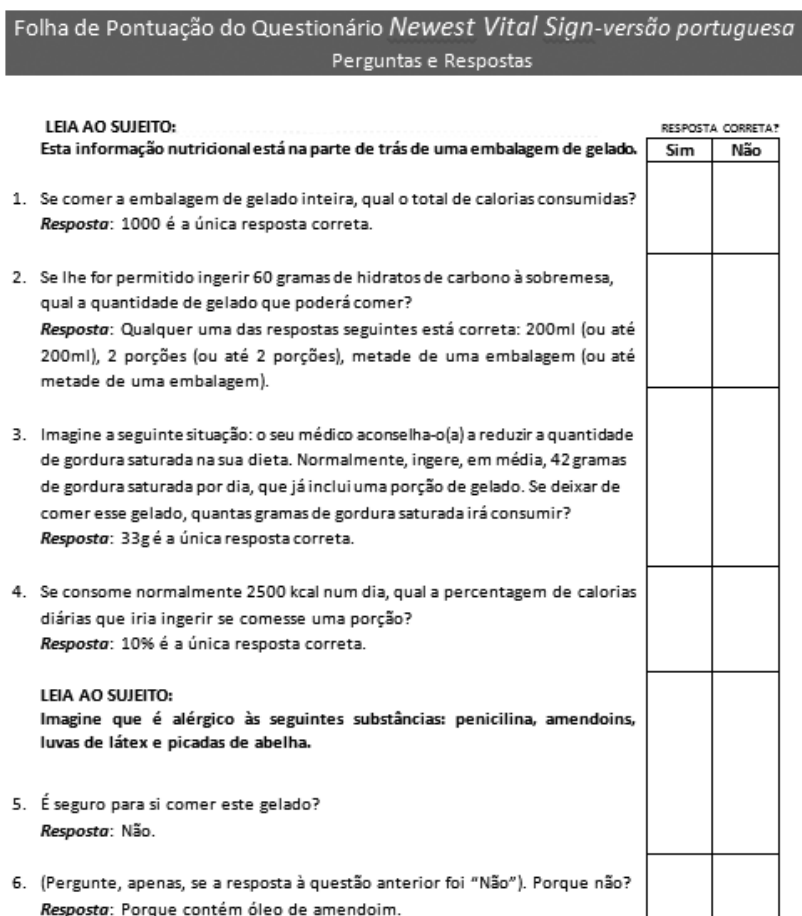

4. Se consome normalmente $2500 \mathrm{kcal}$ num dia, qual a percentagem de calorias diárias que iria ingerir se comesse uma porção? Resposta: $10 \%$ é a única resposta correta.

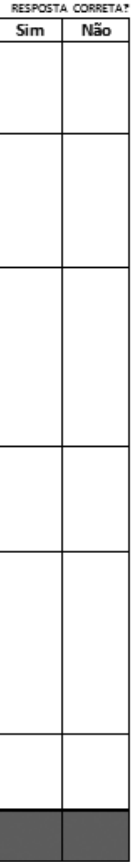

Pontuacão de 0-1 sugere alta probabilidade (50\% ou mais) de literacia limitada. Pontuação de 2-3 indica a possibilidade de literacia limitada. Pontuação de 4-6 quase sempre indica literacia adequada. 


\section{Estudo de validação}

\section{Amostra}

A caracterização demográfica e clínica dos participantes, assim como os estilos de vida/ comportamentais (dieta saudável e atividade física) e o IMC estão indicados na Tabela 1.

Na amostra total, 85,6\% dos indivíduos são mulheres, com uma média de idades de 27,5 anos (DP = 17,5). No que diz respeito a estilos de vida, $25,6 \%$ dos indivíduos estudados ingerem diariamente cinco porções de fruta e/ou vegetais, $72,5 \%$ ingerem menos de cinco porções de fruta e/ou vegetais e

Tabela 1

\section{Caracterização da amostra}

1,9\% não incluem na sua alimentação diária nem fruta nem vegetais. Relativamente à atividade física, 26,3\% da amostra pratica pelo menos 30 minutos por dia de exercício físico de intensidade moderada, 46,6\% menos de 30 minutos diários e para 27,1\% dos indivíduos o exercício físico não faz parte da sua rotina diária. Em termos de IMC, 85,0\% da amostra estudada apresenta valores entre 18,5 e $24,9 \mathrm{Kg} / \mathrm{m}^{2}$ (ideal), 11,6\% distribui-se no nível de risco intermédio (25,0 - 29,9), enquanto 3,4\% está em risco elevado, com um valor igual ou superior a $30 \mathrm{~kg} / \mathrm{m}^{2}$ (Amostra Total, $\mathrm{M}=22,15, \mathrm{DP}=3,07$ ).

\begin{tabular}{|c|c|c|c|c|c|c|}
\hline & \multicolumn{2}{|c|}{$\begin{array}{l}\text { Amostra } 1 \\
(\mathrm{~N}=456)\end{array}$} & \multicolumn{2}{|c|}{$\begin{array}{c}\text { Amostra } 2 \\
(\mathrm{~N}=71)\end{array}$} & \multicolumn{2}{|c|}{$\begin{array}{c}\text { Total } \\
(\mathrm{N}=527)\end{array}$} \\
\hline & Média & DP & Média & $\mathrm{DP}$ & Média & $\mathrm{DP}$ \\
\hline \multirow[t]{2}{*}{ Idade } & 20,89 & 4,43 & 69,82 & 9,16 & 27,48 & 17,54 \\
\hline & $\mathrm{N}$ & $\%$ & $\mathrm{~N}$ & $\%$ & $\mathrm{~N}$ & $\%$ \\
\hline \multicolumn{7}{|l|}{ Género } \\
\hline Feminino & 397 & 87,1 & 54 & 76,1 & 451 & 85,6 \\
\hline Masculino & 59 & 12,9 & 17 & 23,9 & 76 & 14,4 \\
\hline \multicolumn{7}{|l|}{ Dieta saudável } \\
\hline 5 porções de fruta ou vegetais por dia (ideal) & 104 & 23,0 & 30 & 42,3 & 134 & 25,6 \\
\hline Menos de 5 porções de fruta ou vegetais por dia (risco intermédio) & 340 & 75,2 & 39 & 54,9 & 379 & 72,5 \\
\hline Não como fruta nem vegetais (risco elevado) & 8 & 1,8 & 2 & 2,8 & 10 & 1,9 \\
\hline \multicolumn{7}{|l|}{ Atividade física } \\
\hline Pelo menos 30 minutos por dia (ideal) & 107 & 23,7 & 30 & 42,9 & 137 & 26,3 \\
\hline Menos de 30 minutos por dia (risco intermédio) & 215 & 47,7 & 28 & 40,0 & 243 & 46,6 \\
\hline Não faz parte da rotina diária (risco elevado) & 12,9 & 28,6 & 12 & 17,1 & 141 & 27,1 \\
\hline \multicolumn{7}{|l|}{ IMC $\left(\mathrm{kg} / \mathrm{m}^{2}\right)$} \\
\hline Ideal $(18,5-24,9)$ & 407 & 89,8 & 39 & 54,2 & 446 & 85,0 \\
\hline Risco intermédio $(25,0$ - 29,9) & 41 & 9,1 & 20 & 27,8 & 61 & 11,6 \\
\hline Risco elevado (30 ou superior) & 5 & 1,1 & 13 & 18,1 & 18 & 3,4 \\
\hline
\end{tabular}

No que diz respeito ao nível de LS avaliado pelo NVS-PT, 10,3\% dos participantes apresentou alta probabilidade de LS limitada, 13,7\% possibilidade de LS limitada e 76,0\% níveis adequados. Na amostra total, a pontuação média do NVS-PT (respostas certas) foi de 4,44 (DP $=1,70)$ e a da SES 76,0 (DP $=15,3)$ (alfa de Cronbach $=0,89$ ).

\section{Fidelidade}

O alfa de Cronbach foi de 0,67 para o total dos itens, 0,67 para a subescala 1 e 0,83 para a subescala 2 , revelando que o NVS-PT tem consistência interna aceitável. As correlações item-total corrigidas variaram entre 0,31 e 0,45 (Tabela 2) e as correlações inter-itens foram positivas e estatisticamente significativa (Tabela 3). 
Tabela 2

Análise de Fidelidade

\begin{tabular}{lccc}
\hline Item & $\mathrm{M}(\mathrm{DP})$ & Correlação Item-total & Alfa com item eliminado \\
\hline Item 1 & $0,85(0,35)$ & 0,39 & 0,64 \\
Item 2 & $0,81(0,39)$ & 0,42 & 0,63 \\
Item 3 & $0,83(0,37)$ & 0,45 & 0,62 \\
Item 4 & $0,53(0,50)$ & 0,31 & 0,68 \\
Item 5 & $0,85(0,35)$ & 0,40 & 0,63 \\
Item 6 & $0,76(0,42)$ & 0,49 & 0,60 \\
\hline
\end{tabular}

Tabela 3

Correlações inter-itens

\begin{tabular}{lcccccc}
\hline & Item 1 & Item 2 & Item 3 & Item 4 & Item 5 & Item 6 \\
\hline Item 1 & - & $0,360^{* *}$ & $0,352^{* *}$ & $0,348^{* *}$ & $0,299^{* * *}$ & $0,230^{* *}$ \\
Item 2 & $0,360^{* *}$ & - & $0,465^{* *}$ & $0,272^{* *}$ & $0,287^{* *}$ & $0,235^{* *}$ \\
Item 3 & $0,352^{* *}$ & $0,465^{* *}$ & - & $0,301^{* *}$ & $0,264^{* *}$ & $0,242^{* *}$ \\
Item 4 & $0,348^{* *}$ & $0,272^{* *}$ & $0,301^{* *}$ & - & $0,181^{* *}$ & $0,184^{* *}$ \\
Item 5 & $0,299^{* *}$ & $0,287^{* *}$ & $0,264^{* *}$ & $0,181^{* *}$ & - & $0,730^{* *}$ \\
Item 6 & $0,230^{* *}$ & $0,235^{* *}$ & $0,242^{* *}$ & $0,184^{* *}$ & $0,730^{*}$ & - \\
\hline
\end{tabular}

Correlações de Pearson; ** p $<0,001$

\section{Validade de construto}

$\mathrm{Na}$ análise fatorial com rotação varimax, duas subescalas explicam na totalidade $60,97 \%$ da variância, no questionário de 6-itens. Os domínios refletem dois aspetos da LS funcional: aptidões de cálculo e prosa (fator 1) e aptidões no domínio de segurança do consumidor (fator 2) (Tabela 4), confirmada pela associação das subescalas (Tabela 5).

Tabela 4

Resultados da análise exploratória por fator (rotação varimax): Peso dos componentes/itens de cada fator

\begin{tabular}{lcc}
\hline Item & 1 & 2 \\
\hline Item 1 & 0,655 & 0,113 \\
Item 2 & 0,705 & 0,140 \\
Item 3 & 0,732 & 0,137 \\
Item 4 & 0,639 & 0,012 \\
Item 5 & 0,061 & 0,935 \\
Item 6 & 0,202 & 0,904 \\
\hline \% Variância explicada & 31,94 & 29,04 \\
\hline \% Variância acumulada & 31,94 & 60,98 \\
\hline Alfa de Cronbach & 0,67 & 0,83 \\
\hline
\end{tabular}

Kaise-Meyer-Olkin $=0,639$

Tabela 5

Correlações entre as subescalas e a escala total $(N=534)$

\begin{tabular}{lccc}
\hline & 1 & 2 & Total scale \\
\cline { 2 - 4 } Subescala Aptidões de cálculo e prosa (1) & - & & \\
Subescala Aptidões no domínio de segurança do consumidor (2) & $0,285^{* *}$ & - & \\
NVS-PT (Escala Total) & $0,906^{* *}$ & $0,692^{* *}$ & - \\
\hline
\end{tabular}

Pearson's correlations; ** $\mathrm{p}<0,001$ 
A validade de construto também foi apreciada considerando os resultados dos testes de hipóteses de que o NVS-PT estará positivamente associado com melhores pontuações de auto-eficácia, melhores estilos de vida e negativamente com a idade, uma vez que se pressupõe que pessoas mais velhas teriam maior probabilidade de literacia limitada.

O suporte para a validade convergente-divergente foi demonstrada por associações entre o NVS-PT e as suas subescalas com o IMC, a idade e a autoeficácia. Quanto à idade, foram encontradas associações negativas estatisticamente significativas com a escala total $(r=-0,599, p<0,001)$, subescala $1(r$ $=-0,535, p<0,001)$ e subescala $2(r=-0,241, p$ $<0,001)$; foram observadas associações positivas estatisticamente significativas entre a escala total $(r$ $=0,291, p<0,001)$, a subescala de $1(r=0,319$, $p<0,001)$ e a subescala $2(r=0,109, p<0,05)$ e a autoeficácia; finalmente, associações negativas estatisticamente significativas entre escala total $(r$ $=-0,251, p<0,001)$ e a subescala $1(r=-0,214, p$ $<0,001)$ e o IMC. Associações não significativas foram encontradas com os estilos de vida.

A validade discriminante da NVS-PT foi ainda avaliada comparando as diferenças entre participantes com idade igual ou superior a 65 anos de idade $(n=50)$ e com menos de 65 anos de idade, respetivamente, 1,76 e 4,76 (intervalo 0-6): escala total ( $t=14,44$, $p<0,001)$, subescala $1(t=12,58, p<0,001)$ e subescala $2(t=6,41, p<0,001)$.

\section{Discussão}

O presente estudo diz respeito ao processo de tradução para a língua portuguesa e adaptação cultural do NVS, evidenciando a fidelidade e validade do referido instrumento.

No processo de tradução e adaptação cultural não houve dificuldades significativas, tendo sido obtido como resultado final uma versão inteligível do NVS, em língua portuguesa. Weiss et al. (2005) também reportaram resultados similares relativos ao processo de tradução e adaptação cultural da versão espanhola do NVS.

O valor satisfatório do coeficiente de alfa de Cronbach para as duas subescalas e para a escala total, bem como dos coeficientes item-total para os seis itens, confirmou a consistência interna das subescalas do NVS-PT, com correlação entre os seus itens. Comparando este estudo com o de Weiss et al. (2005) em que foi obtido um coeficiente de alfa de Cronbach igual a 0,76 e 0,69 respetivamente para a versão original em língua inglesa e para a versão em espanhol (administrada a nativos de língua inglesa e nativos de língua espanhola, ao nível dos cuidados de saúde primários), os resultados foram semelhantes.

Porque a promoção da LS ocorre num contexto mais alargado, faz sentido a sua associação a outras medidas tais como a autoeficácia e estilos de vida. No presente estudo constatou-se que níveis baixos de LS correlacionam-se com baixa autoeficácia, assim como com pior IMC, representando um aspeto importante a ter em conta em estratégias de promoção da saúde (Zarcadoolas, Pleasant, \& Greer, 2006).

Mais ainda e em consonância com Barrett et al. (2008), confirmou-se que nos idosos é maior a probabilidade de LS limitada. As mulheres pontuaram mais alto do que os homens. No entanto, esta diferença não foi estatisticamente significativa, contrariamente ao estudo de Peerson e Saunders (2009).

Pode assim afirmar-se que o NVS-PT tem potencial para ser usado na identificação dos indivíduos com baixos níveis de LS e, assim, beneficiarem de estratégias de intervenção.

Revela-se também elevada a aplicabilidade do NVS-PT como um instrumento standard para o incremento dos níveis de LS, bem como para facilitar a comunicação utente-profissional de saúde, ao nível dos cuidados de saúde primários. Em ambiente clínico, o NVS-PT pode ser usado para fazer uma estimativa global das aptidões em LS funcional, com o objetivo de apoiar a tomada de decisões clínicas, bem como no planeamento de intervenções de promoção da saúde, quer a título individual quer em populações específicas.

O teste NVS-PT é fácil de usar; o tempo necessário para concluí-lo entre os participantes neste estudo variou de três a seis minutos.

Este estudo sugere que instrumentos como o NVSPT podem ser ferramentas adicionais para avaliar a LS, e nesse sentido poder potenciar a prevenção da doença e a obtenção de melhores resultados em saúde, objetivando a melhoria da comunicação entre doentes e profissionais de saúde em ambientes clínicos ou servir de suporte ao planeamento em promoção da saúde (Institute of Medicine, 2009). 


\section{Limitaçóes do estudo}

Algumas limitações do presente estudo devem ser reconhecidas, nomeadamente o facto de a amostra estudada não poder ser vista como representativa da população portuguesa. Também, embora existam evidências na literatura da existência de associações entre dieta saudável, atividade física e LS, o nosso estudo não as confirmou.

\section{Conclusão}

A versão portuguesa do NVS resultante deste estudo evidencia propriedades psicométricas comparáveis às de outras versões do NVS.

O NVS-PT revelou ser adequado como medida do conceito de LS na população portuguesa. Os níveis aceitáveis de consistência interna do NVS-PT sugerem a sua utilidade na monitorização da LS ao longo do tempo, quer na avaliação do nível basal quer na avaliação de resultados de estratégias de intervenção. Contudo, mais estudos são necessários para confirmar o seu poder de resposta.

Apesar de existirem outros instrumentos de avaliação da LS, a maioria requer um tempo significativo para a sua administração e análise. O NVS foi construído para possibilitar uma administração simples e fácil e poder assim ser usado por si só, ou acoplado a outras medidas de saúde, como parte de um protocolo padrão de avaliação de saúde ao nível dos cuidados de saúde primários ou como medida de suporte de um plano de promoção da saúde.

\section{Referências bibliográficas}

Bandura, A. (1997). Self-efficacy: The exercise of control. New York, NY: Freeman.

Barrett, G., Smith, S. C., \& Wellings, K. (2004). Conceptualisation, development and evaluation of a measure of unplanned pregnancy. Journal of Epidemiology and Community Health, 58(5), 426-433.

Barrett, S. E., Puryear,J. S., \& Westpheling, K. (2008). Health literacy practices in primary care settings: Examples from the field. The Commonwealth Fund, 81(11). Recuperado de http:// www.commonwealthfund.org/ /media/Files/Publications/ Fund\%20Report/2008/Jan/Health\%20Literacy\%20 Practices\%20in\%20Primary\%20Care\%20Settings\%20\%20 Examples\%20From\%20the\%20Field/Barretthltliteracy practicesprimarycaresettingsexamplesfield_1093\%20pdf.pdf
Berkman, N. D., Davis, T. C., \& McCormack, L. (2010). Health literacy: What is it?, Journal of Health Communication: International Perspectives, 15(Suppl. 2), 9-19. doi:10.1080/ 10810730.2010.499985

Dewalt, D. A., Boone, R. S., \& Pignone, M. P. (2007). Literacy and its relationship with self-efficacy, trust, and participation in medical decision making. American Journal of Health Behaviour, 31(Suppl. 1), S27-35. doi:10.5555/ajhb.2007.31. supp.S27

Eichner, J., \& Dullabh, P. (2011). Accessible health information technology (Health IT) for populations with limited literacy: A guide for developers and purchasers of Health IT . Rockville, MD: Agency for Healthcare Research and Quality.

European Health Literacy Survey Consortium. (2012). Comparative report of health literacy in Eight EU Member States. Recuperado de http://www.health-literacy.eu

Federal Interagency Forum on Aging-Related Statistics. (2008). Older Americans 2008: Key indicators of well-being. Recuperado de http://www.agingstats.gov/agingstatsdotnet/ Main_Site/Data/Data_2008.aspx

Institute of Medicine. (2009). Toward health equity and patientcenteredness: Integrating bealth literacy, disparities reduction, and quality improvement: Workshop summary. Washington, DC: National Academies Press.

Nunnally, J. C., \& Bernstein, I. H. (1994). Psychometric theory. New York, NY: McGrawHill.

Pajares, F. (2002). Overview of social cognitive theory and of self-efficacy. Recuperado de http://www.emory.edu/ EDUCATION/mfp/eff.html

Peerson, A., \& Saunders, M. (2009). Men's health literacy: Advancing evidence and priorities. Critical Public Health, 19(3), 441-456. doi:10.1080/09581590902906229

Ribeiro, J. L. P. (1994). Adaptação do "Self-Perception profile for College Students" à população portuguesa: Sua utilização no contexto da psicologia da saúde. In L. Almeida \& I. Ribeiro (Eds.), Avaliação psicológica: Formas e contextos. Braga, Portugal: Apport.

Scientific Advisory Committee of the Medical Outcomes Trust. (2002). Assessing health status and quality-of-life instruments: Attributes and review criteria. Quality of Life Research, 11(3), 193-205.

Sorensen, K., Van den Broucke, S., Fullam, J., Doyle, G, Pelican, J, Slonska, Z., \& Brand, H. (2012). Health literacy and public health: A systematic review and integration of definitions and models. BMC Public Health, 12, 80. doi:10.1186/1471-2458$12-80$.

Staquet, M. J., Hays, R. D., \& Fayers, P. M. (1998). Quality of life assessment in clinical trials: Methods and practice. Oxford, England: Oxford University Press. 
Weiss, B. D., Mays, M. Z., Martz, W., Castro, K. M., DeWalt, D. A., Pignone, M. P., ... Hale, F. A. (2005). Quick assessment of literacy in primary care: The newest vital Sign. Annals of Family Medicine, 3(6), 514-522. doi:10.1370/afm. 405

White, S. (2008). Assessing the Nation's Health Literacy - Key concepts and findings of the National Assessment of Adult Literacy (NAAL). Recuperado de http://www.ama-assn.org/ resources/doc/ama-foundation/hl_report_2008.pdf
World Health Organization (2009). Background note: Regional Preparatory Meeting on Promoting Health Literacy. Recuperado de http://www.un.org/en/ecosoc/newfunct/pdf/ chinameetinghealthliteracybackgroundpaperv2.pdf

Zarcadoolas, C., Pleasant, A., \& Greer, D. S. (2006). Advancing bealth literacy. A framework for understanding and action. San Francisco, CA: John Wiley \& Sons. 